\title{
Coexpression of Two Odorant-Binding Protein Homologs in Drosophila: Implications for Olfactory Coding
}

\author{
Daria S. Hekmat-Scafe, ${ }^{1}$ R. Alexander Steinbrecht, ${ }^{2}$ and John R. Carlson ${ }^{1}$ \\ ${ }^{1}$ Department of Biology, Yale University, New Haven, Connecticut 06520-8103, and ${ }^{2}$ Max-Planck-Institut für \\ Verhaltensphysiologie, D-82319 Seewiesen, Germany
}

\begin{abstract}
Odorant-binding proteins (OBPs) are small soluble proteins present in the aqueous medium surrounding olfactory receptor neurons. Their function in olfaction is still unknown: they have been proposed to facilitate the transit of hydrophobic molecules to olfactory receptors, to deactivate the odorant stimulus, and/or to play a role in chemosensory coding. In this study we examine the genomic organization and expression patterns of two olfactory-specific genes (OS-E and OS-F) of Drosophila melanogaster, the products of which are members of a protein family in Drosophila sharing sequence similarity with moth OBPs. We show that the OS-E and OS- $F$ transcription units are located $<1 \mathrm{~kb}$ apart. They are oriented in the same direction and display a similar intron-exon organization. Expression of
\end{abstract}

both OS-E and OS-F proteins is restricted spatially to the ventrolateral region of the Drosophila antenna. Within this region both OS-E and OS-F proteins are expressed within two different types of sensory hairs: in most, if not all, sensilla trichodea and in $\sim 40 \%$ of the interspersed small sensilla basiconica. We consistently observe that OS-E and OS-F are coexpressed, indicating that an individual sensillum can contain more than one odorant-binding protein. The functional significance of the observed expression pattern and its implications for olfactory coding are discussed.

Key words: odorant-binding protein; Drosophila; sensilla; olfaction; antenna; pheromone-binding protein
In the olfactory systems of terrestrial animals, olfactory receptor neurons extend dendrites into an aqueous phase (vertebrate nasal mucus or insect sensillar lymph) that protects them from desiccation (Farbman, 1992; Hildebrand, 1995). It is not known how hydrophobic odorant molecules traverse these aqueous barriers to reach the odorant receptors. However, both vertebrate olfactory mucus and insect sensillar lymph contain families of small, abundant water-soluble odorant-binding proteins (OBPs) that have been proposed to shuttle hydrophobic odorants to and/or from odorant receptors (Pelosi, 1994; Pelosi and Maida, 1995; Prestwich et al., 1995).

In addition to potentially transporting odorant molecules, OBPs also may play a role in olfactory coding. Multiple related OBPs have been found within the same vertebrate or moth species (Pelosi, 1994; Pelosi and Maida, 1995). These different OBPs may recognize different odorants: two OBPs expressed in the moth Antheraea pernyi have been shown to display different binding preferences for two moth pheromones (Du and Prestwich, 1995; Prestwich et al., 1995). If OBPs with different odorant specificities are restricted to different regions of the olfactory system, they might influence which odorants have access to the underlying olfactory receptors in these regions (Vogt et al., 1991).

Moths contain two classes of OBPs: pheromone-binding proteins (PBPs), which bind pheromones in vitro (Vogt and Riddiford, 1981), and general odorant-binding proteins (GOBPs), the ligands of which are unknown (Breer et al., 1990). PBPs and

Received Aug. 28, 1996; revised Dec. 17, 1996; accepted Dec. 23, 1996.

This work was supported by a National Institute of Deafness and Other Communication Disorders grant to J.C. (R01 DC02174-10) and a research service award to D.H. (DC00139). We are grateful to Barbara Müller for her skillful assistance at the immunocytochemical studies. We thank Michael McKenna, Charles Scafe, and Michael Laue for helpful discussions.

Correspondence should be addressed to Dr. Carlson at the above address.

Copyright (C) 1997 Society for Neuroscience 0270-6474/97/171616-09\$05.00/0
GOBPs are present at high concentrations in the sensillar lymph surrounding the dendrites of receptor cells tuned to pheromones or general odorants, respectively (Laue et al., 1994; Steinbrecht et al., 1992, 1995).

In Drosophila, a family of genes has been isolated, the predicted products of which share sequence similarity with moth OBPs and the transcripts of which are restricted to various subregions of the olfactory system (McKenna et al., 1994; Pikielny et al., 1994). We have concentrated on two such olfactory-specific genes $(O S-E$ and $O S-F)$, the products of which are structurally similar (68\% amino acid identity). $O S-E$ and $O S-F$ transcripts both are observed specifically in the ventrolateral region of the antenna (McKenna et al., 1994; Pikielny et al., 1994). This pattern is similar to the distribution of one class of olfactory sensory hairs, the sensilla trichodea (Venkatesh and Singh, 1984). The ventrolateral region of the Drosophila antenna also contains a number of small sensilla basiconica, although most sensilla basiconica are located in the reciprocal dorsomedial region of the antenna (Venkatesh and Singh, 1984; Stocker, 1994). Drosophila sensilla basiconica respond to general odorants (Siddiqi, 1987), as has been observed in other insects.

In this study, we show that the $O S-E$ and $O S-F$ genes are located $<1 \mathrm{~kb}$ apart and contain a similar intron-exon organization. We demonstrate that expression of both OS-E and OS-F proteins is restricted spatially to two distinct types of olfactory hairs located in the ventrolateral region of the antenna: most, if not all, sensilla trichodea and a subset of the small sensilla basiconica. Most interestingly, our immunocytochemical EM studies reveal that OS-E and OS-F are coexpressed, in the sense that all sensilla examined that express one also express the other. Thus, an individual sensillum can contain more than one odorantbinding protein. This finding has potential implications for the roles of odorant-binding proteins in olfactory coding. 
A

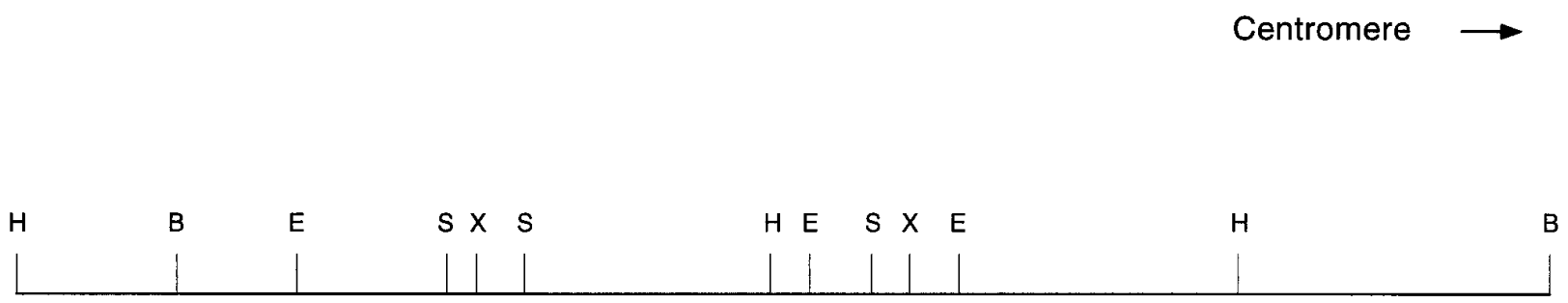

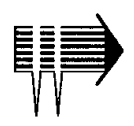

OS-E

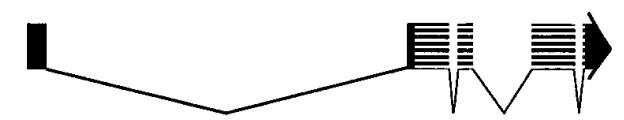

OS-F (1)
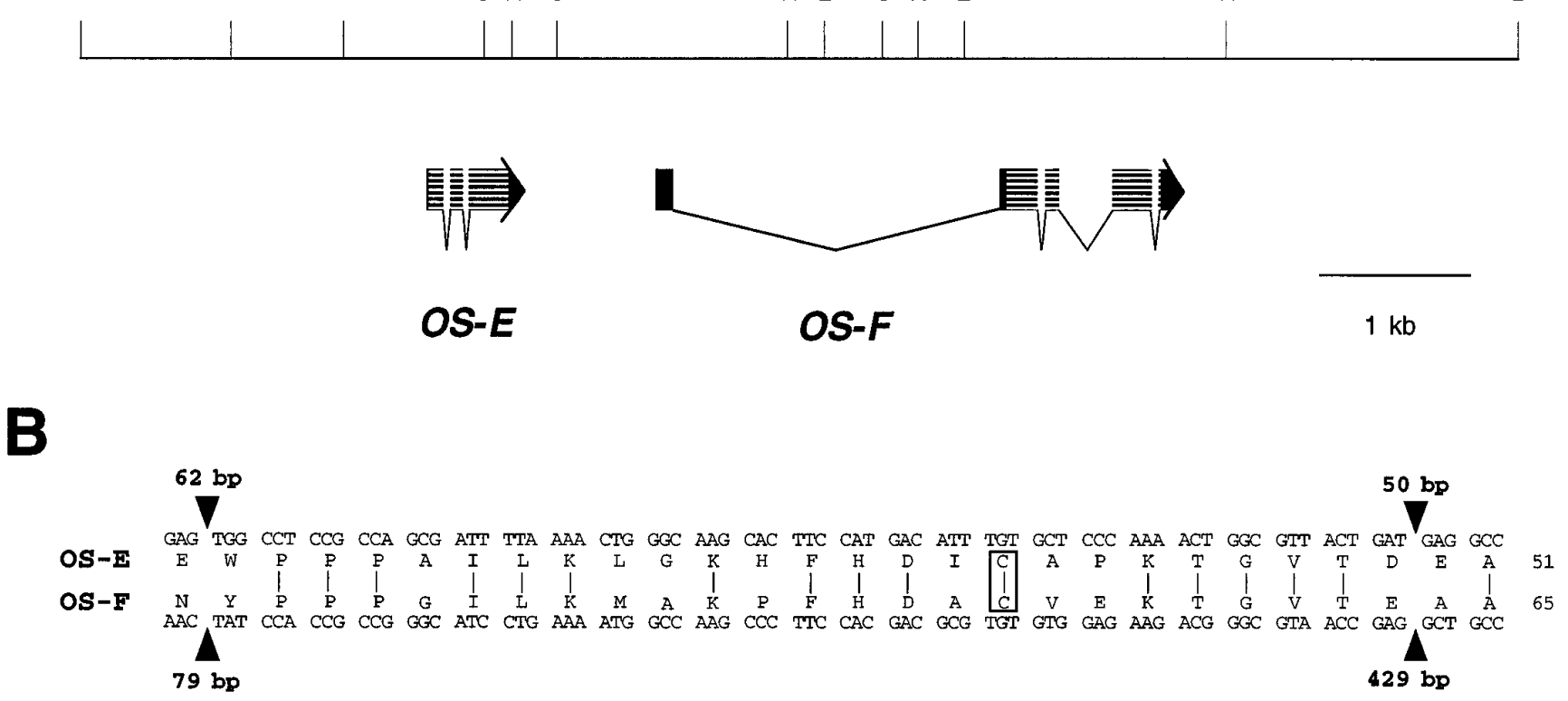

Figure 1. Genomic organization of $O S-E$ and $O S-F$ genes. $A$, Genomic organization of the $O S-E$ and $O S-F$ transcription units. Both genes are transcribed from left to right, as indicated by the arrows. Exons of cDNAs are indicated by thick boxes, and introns are shown as thin lines below these boxes. Coding sequences are shown as striated boxes; noncoding sequences are indicated by solid boxes. $B$, Sequence of a portion of $O S-E$ and $O S-F$ showing the location of two introns found at identical positions in each gene. The sequence alignment between $O S-E$ and $O S-F$ corresponds to amino acids $24-51$ of $O S$ - $E$ and 38-65 of $O S-F$. The first of six conserved cysteine residues is boxed. Intron sizes and insertion sites (arrowheads) are indicated. Note that the two introns in $O S-E$ (62 and $50 \mathrm{bp}$ in size) are located at positions corresponding to those of two introns in $O S-F$ (79 and 429 bp in size).

\section{MATERIALS AND METHODS}

Mapping the OS-E and OS-F genomic region. The $\lambda \mathrm{GE} 1$ and $\lambda \mathrm{GE} 3$ clones, which carry $O S-E$ and $O S-F$ genomic DNA, were isolated (McKenna et al., 1994) from a Canton-S genomic library in $\lambda$ EMBL3 (kindly provided by I. Dawson, Yale University) with an $O S-E$ cDNA probe. Plasmid subclones of portions of these genomic DNAs were prepared as follows: A $4.7 \mathrm{~kb}$ HindIII fragment of $\lambda \mathrm{GE} 1$ (containing $O S-E$ as well as the first

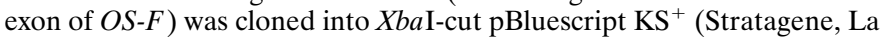
Jolla, CA) to create pDH58; a $1.9 \mathrm{~kb}$ SalI fragment of $\lambda \mathrm{GE} 1$ (containing the first exon of $O S-F$ ) was cloned into Bam HI-cut pBluescript $\mathrm{KS}^{+}$to create pDH59; and a $12 \mathrm{~kb}$ SalI fragment of $\lambda \mathrm{GE} 3$ (containing the last three exons of $O S-F$ ) was cloned into Bam HI-cut pBluescript $\mathrm{KS}^{+}$to create $\mathrm{pDH} 60$. In all cases, the recessed termini of the vector and insert were partially filled in with Klenow and the first two dNTPs to create cohesive termini, as described (Hung and Wensink, 1984). Restriction mapping of pDH58-60 was performed by standard procedures (Sambrook et al., 1989).

A series of $5^{\prime}$ and $3^{\prime}$ primers was designed to span the $O S-E$ and $O S-F$ cDNA sequences (McKenna et al., 1994) (GenBank accession numbers U02543 and U02542, respectively). These primers were used to amplify $O S-E$ and $O S-F$ genomic sequences (from pDH58 or pDH59-60, respectively) or cDNA sequences [from pDH50 or FF4 (McKenna et al., 1994), respectively], using PCR. PCR conditions were as follows: denaturation at $94^{\circ} \mathrm{C}$ for $5 \mathrm{~min}$, followed by 35 cycles of $48^{\circ} \mathrm{C}$ for $1 \mathrm{~min}, 72^{\circ} \mathrm{C}$ for $2 \mathrm{~min}$, and $94^{\circ} \mathrm{C}$ for $45 \mathrm{sec}$, and then one cycle of $48^{\circ} \mathrm{C}$ for $3 \mathrm{~min}$ and $72^{\circ} \mathrm{C}$ for 10 min. PCR reaction products were analyzed by $1.2 \%$ agarose/Tris borate EDTA gel electrophoresis. In cases in which PCR amplification of the corresponding genomic and cDNA clones produced fragments of identical size, we concluded that no intron was present in the corresponding stretch of genomic DNA. When the PCR product produced from a genomic template was larger than one made from the cDNA template, we concluded that one or more introns were present in the stretch of genomic DNA. Both strands of the corresponding stretch of genomic DNA were subjected to dideoxynucleotide sequence analysis with the appropriate PCR primers and a Sequenase 2.0 kit (Stratagene), according to the manufacturer's directions. In each case, the intron insertion site was determined from both directions. Additional genomic sequence analysis of portions of the long $5^{\prime}$ untranslated region of $O S-F$ (present in pDH59) was performed to ensure that no additional introns were present. Sequence analysis of the $O S-E$ and $O S-F 5^{\prime}$ upstream regions in pDH58 and pDH60, respectively, was performed in an analogous manner. Additional sequencing of the third $O S-F$ intron and the intervening region between $O S-E$ and $O S-F$ was done by the W. M. Keck Foundation Biotechnology Resource Laboratory at Yale University. The $O S-E$ and $O S-F$ genomic sequences have been submitted to GenBank (U81502 and U81503, respectively).

Two bacteriophage P1 (DS00431 and DS06666) carrying $\sim 80 \mathrm{~kb}$ inserts of Drosophila genomic DNA (Smoller et al., 1991), which crosshybridized to a hexamer-labeled (Feinberg and Vogelstein, 1983) OS-F probe, were identified by Southern hybridization (Sambrook et al., 1989). Bacteriophage DNAs were prepared as described (Pierce and Sternberg, 1992) from a series of isolates of the Drosophila P1 library known to map to chromosomal region 83CD (Berkeley Drosophila Genome Project) and were bound to nitrocellulose with a Hybri-Slot filtration manifold (BRL, Bethesda, MD), essentially as described by the manufacturer. The position and orientation of the $O S-E$ and $O S-F$ genomic locus on a deduced restriction map encompassing DS06666 and a portion of DS00431 were used to determine the orientation of the $O S-E$ and $O S-F$ locus relative to the centromere of Chromosome III.

Production and purification of His-tagged $O S-E$ and $O S-F$ proteins. A DNA fragment corresponding to mature $O S-E$ carrying a $6 x$ His tag at its $\mathrm{C}$ terminus was produced by PCR amplification of pDH50 (McKenna et al., 1994) with the 5' primer E9 (5' GGG ATT CCA TAT GCA GGA ACC AAG GCG CGA TGG) and the 3' primer E5 (5' GCT CTA GAT 


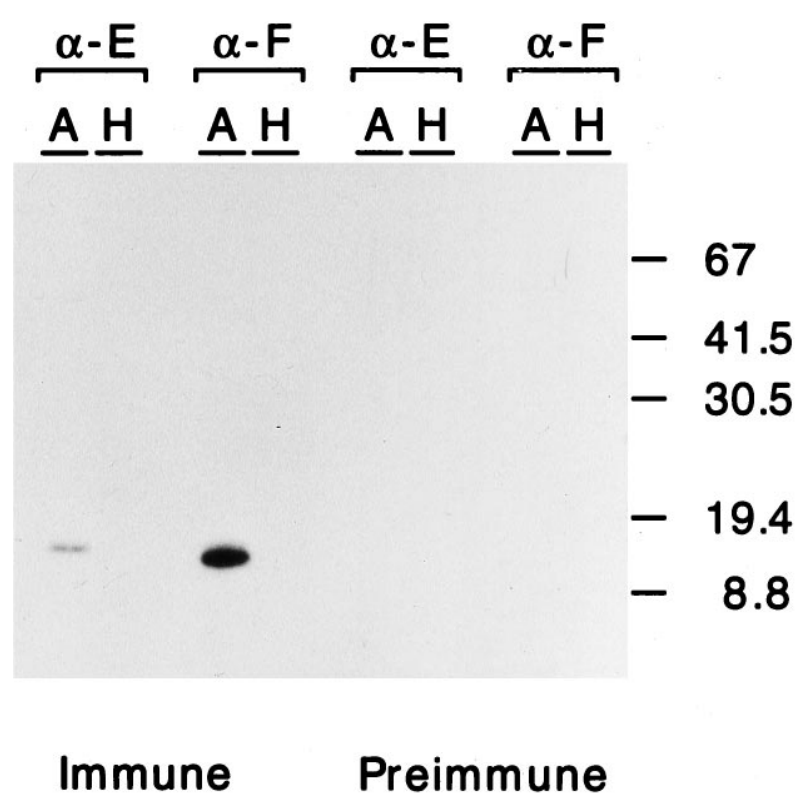

Figure 2. Western analysis of OS-E and OS-F proteins in Drosophila antennae and heads. Body parts were collected from equal numbers of male and female Drosophila. Fractions of the resulting extracts corresponding to 25 antennae $(A)$ or $1 \frac{1}{2}$ heads depleted of antennae $(H)$ were subjected to $15 \%$ SDS-PAGE and transferred to nitrocellulose. Primary antisera were either affinity-purified anti-OS-E $(\alpha-E)$, affinity-purified anti-OS-F $(\alpha-F)$, or preimmune sera from the corresponding mice. Positions of molecular weight markers are indicated on the right.

TAA TGG TGA TGG TGA TGG TGG ACC AAA AAG TAG TGG ACA GG). One corresponding to mature $O S-F$ carrying a $6 x H$ is tag at its $\mathrm{C}$ terminus was produced by PCR amplification of FF4 (McKenna et al., 1994 ) with the 5' primer F7 (5' GGA ATT CCA TAT GCT GAT CCT GCC GCC GGC TGC) and the $3^{\prime}$ primer F4 (5' GCT CTA GAT TAA TGG TGA TGG TGA TGG TGC GGC AAG AAG TAG TGC TTG G). Vent DNA polymerase (New England Biolabs, Beverly, MA) was used as recommended by the manufacturer. PCR conditions were as follows: denaturation at $94^{\circ} \mathrm{C}$ for $5 \mathrm{~min}$, followed by 15 cycles of $55^{\circ} \mathrm{C}$ for $45 \mathrm{sec}$, $72^{\circ} \mathrm{C}$ for $1 \mathrm{~min}$, and $94^{\circ} \mathrm{C}$ for $45 \mathrm{sec}$, and then one cycle of $55^{\circ} \mathrm{C}$ for $45 \mathrm{sec}$ and $72^{\circ} \mathrm{C}$ for $5 \mathrm{~min}$. Tagged $O S-E$ and $O S-F$ fragments were subcloned into the T7-SS expression plasmid (a kind gift of S. J. Smerdon, Yale University) to create pDH97 and pDH98, respectively.

Cultures of Escherichia coli strain BL21 ( $\lambda$ DE3) carrying either pDH97 or pDH98 were harvested after $3 \mathrm{hr}$ of induction with $4 \mathrm{~mm}$ isopropyl thiogalactoside (IPTG). Frozen cell pellets were resuspended in $10 \mathrm{ml}$ of buffer A (6 mM guanidinium $\mathrm{HCl}, 0.1 \mathrm{M}$ Na-phosphate, and $0.01 \mathrm{M}$ Tris, $\mathrm{pH}$ 8.0) containing $10 \mathrm{~mm} \beta$-mercaptoethanol ( $\beta$-ME), and His-tagged OS-E and OS-F proteins were isolated on Ni-NTA resin (Qiagen, Hilden, Germany) under denaturing conditions as recommended by the manufacturer, except that a batch procedure (Nonet et al., 1993) was used. Proteins were renatured under oxidizing conditions used successfully for Antheraea polyphemus PBP (Prestwich, 1993), except that dialysis was concurrent with renaturation. Briefly, $5.5 \mathrm{ml}$ of each eluate was brought to $14.5 \mathrm{~mm}$ cysteine, $\mathrm{pH} 8$, and incubated on a room temperature rotator for $10 \mathrm{~min}$ to oxidize the residual $\beta$-ME. Then the resulting samples were dialyzed overnight at $4^{\circ} \mathrm{C}$ in $0.1 \mathrm{M}$ Tris, $5 \mathrm{~mm}$ cysteine, and $0.02 \%$ sodium azide $\left(\mathrm{NaN}_{3}\right), \mathrm{pH} 8$. The samples were concentrated to $\sim 1 \mathrm{mg} / \mathrm{ml}$ in PBS, pH 8, using Centriprep-10 filters (Amicon, Beverly, MA), as described by the manufacturer.

Production and affinity purification of anti-E and anti-F antisera. For production of each antiserum, four mice were immunized with $50 \mu \mathrm{g}$ of the corresponding His-tagged protein, boosted with an additional $50 \mu \mathrm{g}$ of protein after 3 and 5 weeks, and then boosted with $10 \mu \mathrm{g}$ of protein every 4 weeks thereafter. The protein preparations were mixed with equal volumes of Complete Freund's adjuvant for the initial immunizations and of Incomplete Freund's adjuvant for the subsequent boosts. Aliquots (100 $\mu \mathrm{l})$ of anti-E and anti-F antisera were affinity-purified on $100 \mu \mathrm{g}$ of the corresponding His-tagged protein coupled to Reacti-Gel 6x beads (Pierce) in $0.1 \mathrm{~m}$ Na-borate, $\mathrm{pH} 8.5$, as indicated by the manufacturer.
The affinity purification procedure of Harlow and Lane (1988) was adapted for a microfuge tube; all steps were performed at room temperature. Each serum sample was diluted fivefold with $10 \mathrm{~mm}$ Tris, $\mathrm{pH} 7.5$, and incubated with the corresponding beads for $45 \mathrm{~min}$ on a rotator, compacted $(\sim 0.5 \mathrm{~min}$ at $500 \times g)$, and washed with $10 \mathrm{~mm}$ Tris, $\mathrm{pH} 7.5$, and then with $10 \mathrm{~mm}$ Tris, $\mathrm{pH} 7.5 / 0.5 \mathrm{~mm} \mathrm{NaCl}$. Bound protein was eluted by a $15 \mathrm{~min}$ incubation with $0.1 \mathrm{M}$ glycine, $\mathrm{pH} 2.5$ (corresponding to $2.5 \times$ the original volume of serum), and the eluate was combined immediately with an equal volume of $1 \mathrm{M}$ Tris, $\mathrm{pH} 8.0,100 \mu \mathrm{g} / \mathrm{ml}$ bovine serum albumin, and $0.02 \% \mathrm{NaN}_{3}$.

For preparation of subtracted anti-E antibody, the primary anti-E serum (diluted fivefold with $10 \mathrm{~mm}$ Tris, $\mathrm{pH}$ 7.5) was added to OS-Fcoupled beads and incubated on a rotator for $1.5 \mathrm{hr}$ at room temperature. Then the beads were compacted and the unbound antibody fraction applied to OS-E-coupled beads and affinity-purified as described above, except that bound protein was eluted with a volume of $0.1 \mathrm{M}$ glycine, $\mathrm{pH}$ 2.5 , which corresponded to $12.5 \times$ the original volume of serum. The reciprocal purification scheme was used to prepare the subtracted anti-F antibody. All affinity-purified antibodies were stored at $4^{\circ} \mathrm{C}$.

Western analysis and immunolocalization. The Drosophila strain used for all immunological analyses, D222, is a derivative of Canton S-5 (Helfand and Carlson, 1989) made isogenic for Chromosome III (where $O S-E$ and $O S-F$ are located). Drosophila antennae were collected from equal numbers of males and females after immersion in liquid nitrogen as described by Störtkuhl et al. (1994). SDS-PAGE sample buffer (Sambrook et al., 1989) was added directly to the frozen antennae, the sample heated to $95^{\circ} \mathrm{C}$ for $10 \mathrm{~min}$, and the sample microfuged at top speed for 10 min to pellet cellular debris.

SDS-PAGE and Western transfer to BA83 nitrocellulose (Schleicher \& Schuell, Keene, NH) were done by standard methods (Sambrook et al., 1989). Protein was detected with a Western light chemiluminescent detection system (Tropix, Bedford, MA) essentially as described by the manufacturer, except that blocking was done overnight at $4^{\circ} \mathrm{C}$. Preimmune sera were diluted $1: 1000$, the $5 \times$ more dilute affinity-purified antisera 1:200, and the $25 \times$ more dilute subtracted antisera 1:40.

Immunohistochemistry on Drosophila head sections was performed as described by Raha and Carlson (1994), except that tissue fixation was accomplished by a $1 \mathrm{~min}$ incubation in Histochoice (AMRESCO, Solon, $\mathrm{OH})$, and detection was performed with a Vectastain $\mathrm{ABC}$ elite kit (Vector Labs, Burlingame, Ca) essentially as described by the manufacturer. Preimmune sera were diluted 1:2000 and affinity-purified antisera, 1:200. The secondary antibody, biotinylated anti-mouse $\operatorname{IgG}$ (Vector Labs), was diluted 1:300.

For immunocytochemistry at the electron microscopic level, Drosophila antennae and maxillary palps were cryofixed by plunging the heads with the attached appendages into super-cooled propane at $-180^{\circ} \mathrm{C}$. Then specimens were freeze-substituted in acetone (pure or containing 3\% glutaraldehyde) at $-80^{\circ} \mathrm{C}$, embedded in LR White resin (London Resin) at room temperature and polymerized at $60^{\circ} \mathrm{C}$ (Steinbrecht, 1993). Ultrathin sections were cut with a diamond knife on a Reichert OmU2 ultramicrotome and picked up on Formvar-coated single-hole grids. The affinity-purified anti-E and anti-F antisera were diluted from 1:200 to 1:1000; the corresponding subtracted antibodies were diluted from 1:60 to 1:200. Preimmune sera, used as control, were diluted from 1:200 to $1: 10000$. Goat anti-mouse IgG conjugated with $10 \mathrm{~nm}$ of colloidal gold (BioCell, Cardiff, UK) was used as the secondary antibody and was diluted 1:20. Silver intensification (Danscher, 1981) enlarged the grains to $\sim 40 \mathrm{~nm}$. Further details of the immunocytochemical protocol are described by Steinbrecht et al. (1995).

\section{RESULTS}

\section{$O S-E$ and $O S-F$ genes are located in close proximity to each other}

A detailed analysis of the genomic region encompassing $O S-E$ and $O S-F$ is shown in Figure $1 A$. The $O S-E$ and $O S-F$ transcription units are 0.64 and $3.7 \mathrm{~kb}$, respectively. The two transcription units are oriented in the same direction $\sim 930$ bp apart (Fig. $1 A$ ). Two small introns are present in the $O S-E$ coding region: one of $62 \mathrm{bp}$ between $\mathrm{E}^{24}$ and $\mathrm{W}^{25}$ and another of $50 \mathrm{bp}$ between $\mathrm{D}^{49}$ and $\mathrm{E}^{50}$ (Fig. $1 B$ ). OS-F has three introns in its coding region: one of $79 \mathrm{bp}$ between $\mathrm{N}^{38}$ and $\mathrm{Y}^{39}$, one of 429 bp between $\mathrm{E}^{63}$ and $\mathrm{A}^{64}$, and one of $54 \mathrm{bp}$ between $\mathrm{K}^{149}$ and $\mathrm{H}^{150}$. OS- $F$ has a fourth intron of $\sim 2.2$ 


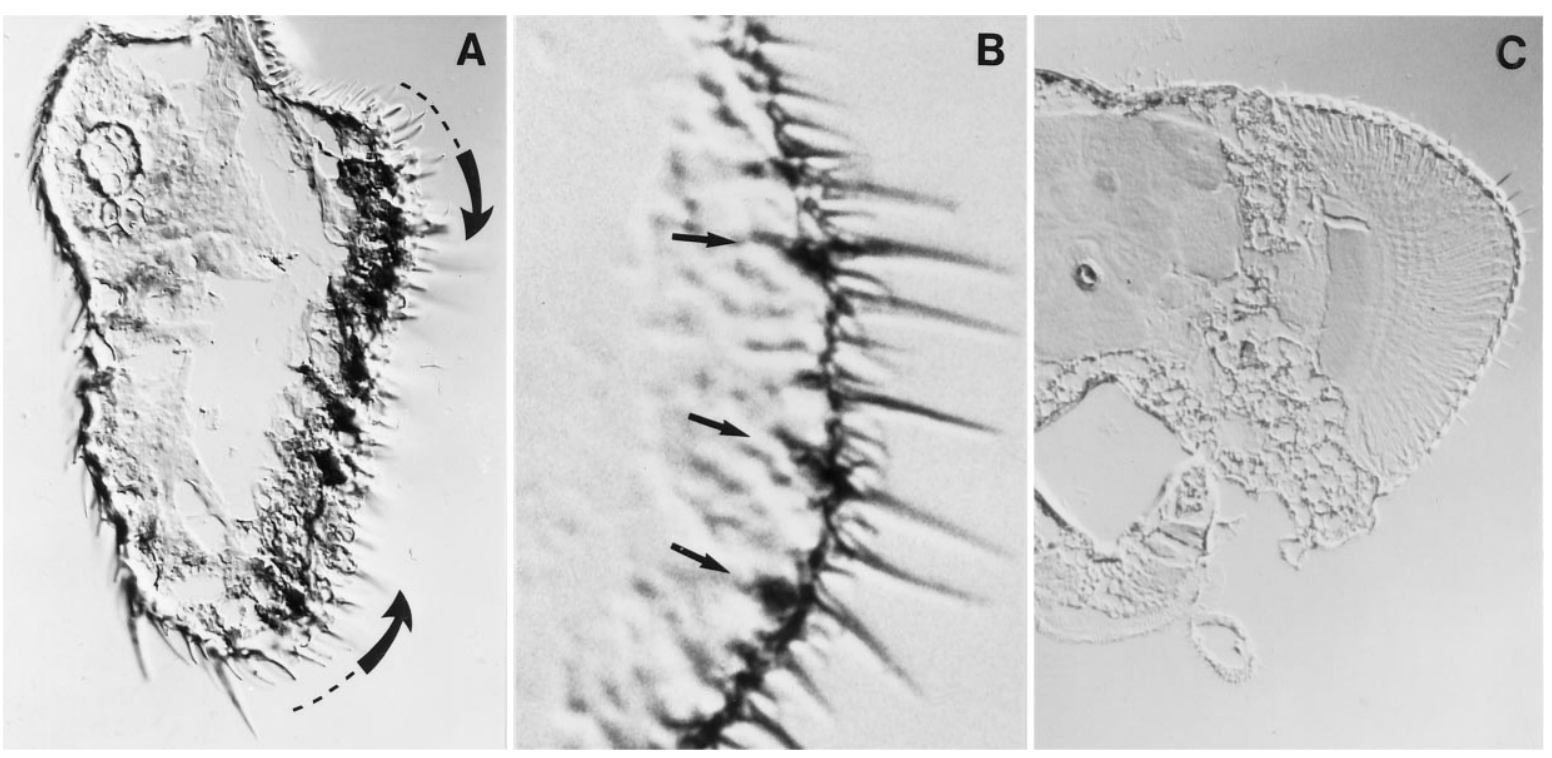

Figure 3. Immunohistochemical staining of OS-E and OS-F in Drosophila antennae. A, Representative immunohistochemical staining of a Drosophila antenna with affinity-purified $\alpha$-E $(1: 200)$. The antenna is oriented such that lateral is right and ventral is down. Strong staining is observed beneath the cuticle in the lateral portion of the antenna. The strongest staining is in the region whose boundaries are indicated by arrows; weaker staining is observed in the area indicated by the dotted lines. Magnification is $200 \times$. B. Representative immunohistochemical staining of a Drosophila antenna with affinity-purified $\alpha-\mathrm{F}$ (1:200). Again, lateral is right and ventral is down. Staining observed beneath three individual sensilla trichodea is indicated by arrows. Magnification is $400 \times$. C, Immunohistochemical staining of a Drosophila head section with affinity-purified $\alpha$-E (1:200). The right half of the head is shown such that lateral is right and ventral is down. Note the absence of discernible specific staining in the brain (left, center to top) and eye (right). Magnification is $50 \times$.

$\mathrm{kb}$ in its $5^{\prime}$ noncoding region. The two introns in $O S-E$ are present at locations identical to those of two of the introns in $O S-F$ (Fig. 1B).

Genomic DNA upstream of the presumptive $O S-E$ and $O S-F$ transcriptional start sites, as determined by $5^{\prime}$ reverse transcriptase amplification of cDNA (McKenna et al., 1994; Pikielny et al., 1994), revealed a TATAAA sequence at -29 relative to the $5^{\prime}$ end of the OS-F cDNA. Although no clear TATA box was seen in the $O S-E$ upstream DNA, the sequence ATAAAA was present at -32 from the $5^{\prime}$ end of the cDNA. Analysis of several hundred nucleotides of genomic DNA sequence upstream of $O S-E$ and $O S-F$ does not reveal extensive sequence similarities between the upstream regions of the two genes. However, the $225 \mathrm{bp}$ upstream of the $O S-E 5^{\prime}$ end contains five precise repeats and one variant repeat (containing one different nucleotide) of the octamer PyCATTTPuPy (data not shown), which may represent a repeated enhancer motif. The 425 bp upstream of the $O S-F 5^{\prime}$ end contains three precise and three variant repeats of the related heptamer CATTTPuPy (data not shown).

Low-stringency Southern hybridization of EcoRI, HindIII, $B a m \mathrm{HI}$, or $X b a \mathrm{I}$-digested genomic DNA with either an $O S-E$ or OS-F cDNA probe revealed only the expected $O S-E$ and $O S-F$ fragments (data not shown), suggesting that there are no other genes closely related to $O S-E / O S-F$ either in this cluster or elsewhere in the Drosophila genome.

\section{OS-E and OS-F are found in two types of sensilla}

Recombinant bacterially expressed proteins corresponding to mature OS-E or OS-F (each with a "6xHis tag" of six Histidine residues at the $\mathrm{C}$ terminus) were used to generate polyclonal anti-E and anti-F antisera in mice. Western analysis revealed that affinity-purified anti-E and anti-F antisera both recognize small proteins expressed in antennae, but not heads (Fig. 2). The sizes of the antennal proteins detected with the anti-E and anti-F antisera were 14.5 and 13.5 , respectively, which are in reasonable agreement with those expected for mature OS-E and OS-F-14.4 and $14.1 \mathrm{kDa}$, respectively (McKenna et al., 1994). Neither preimmune serum demonstrates discernible reactivity with proteins present in either the antennal or head extract. Male and female antennae showed no significant differences in the sizes or abundance of OS-E and OS-F proteins (data not shown).

Immunohistochemical analysis with the affinity-purified anti-E and anti-F antisera shows labeling in the lateral and ventral region of the antenna (Fig. 3A), where their transcripts are also present (McKenna et al., 1994). Labeling often was found beneath individual sensilla trichodea (Fig. $3 B$ ) and, occasionally, sensilla basiconica (data not shown). Although the resolution of this analysis does not allow a precise identification of the cells labeled, we note that the location of the label directly beneath the cuticle corresponds to the position of the sensillar auxiliary cells and the sensillum lymph cavities; neuronal cell bodies are located further below the cuticle (R. A. S., unpublished observations). In some cases staining extends into the sensory hair itself (Fig. $3 B$ ). No discernible staining was observed in the brain or other chemosensory organs (maxillary palps and proboscis) with either the anti-E or anti-F antiserum (Fig. $3 C$ and data not shown). The anti-F antiserum did show some staining in the eye. Because no $O S-F$ transcript is detectable in the eye by RNA in situ hybridization (McKenna et al., 1994) nor OS-F protein in heads by Western (Fig. 2), this staining most likely reflects nonspecific labeling.

The sensillar and subcellular distribution of OS-E and OS-F proteins was examined at higher resolution with immunocytochemical electron microscopy. In the electron microscope, sensilla trichodea can be distinguished from sensilla basiconica by the thicker cuticular wall of the sensilla trichodea. In addition, sensilla trichodea have essentially unbranched outer dendritic segments and a smaller number of pores per unit of surface area, as 

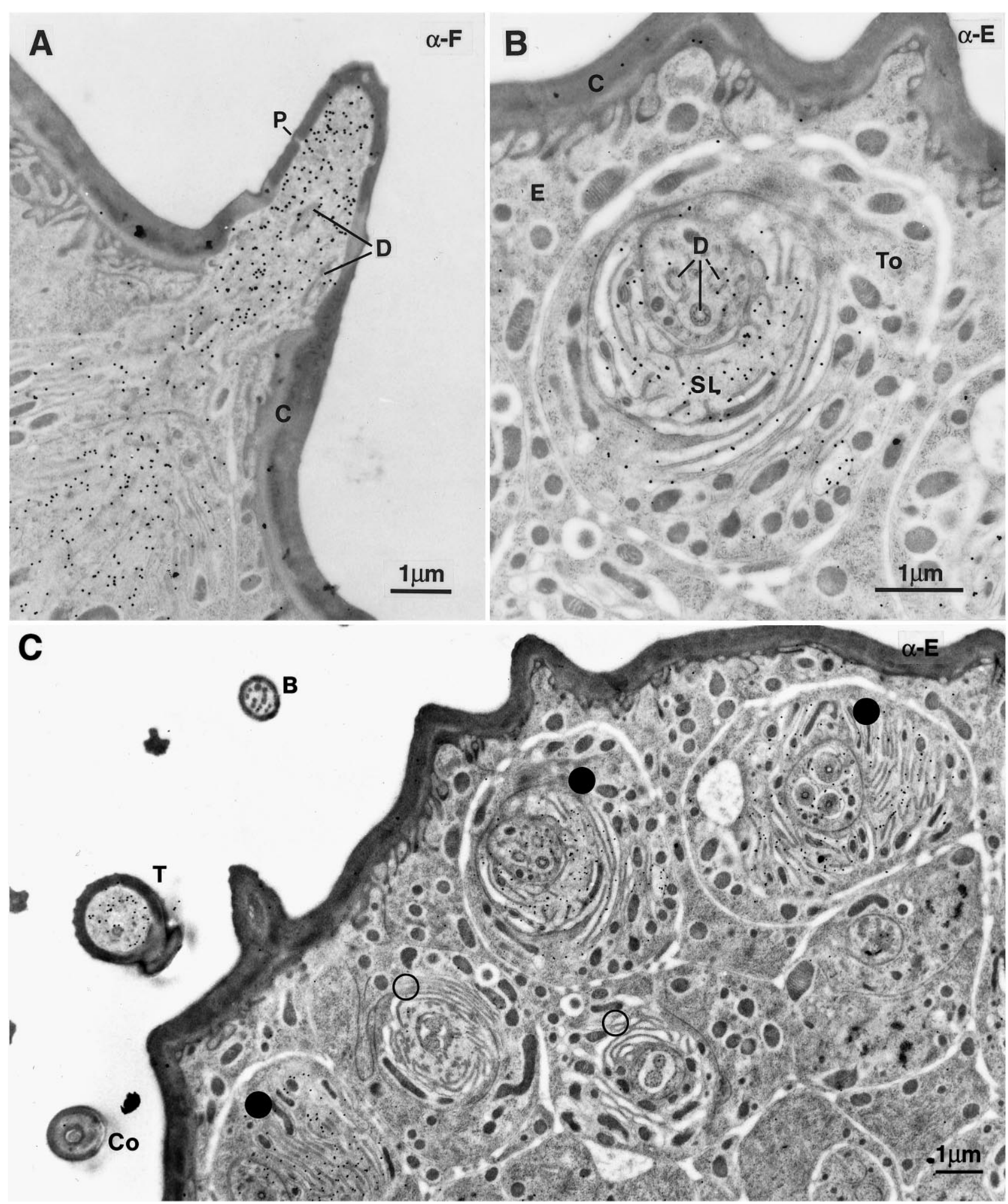

Figure 4. Immunocytochemical localization of OS-E and OS-F in Drosophila olfactory sensilla. $A$, Longitudinal section through the base of a sensillum trichodeum labeled with $\alpha-\mathrm{F}(1: 1000)$. The sensillum lymph in the hair lumen and in the cavity below the hair base is strongly labeled. $P$, Pore; $D$, dendrite, $C$, cuticle. $B$, Cross section of an olfactory sensillum below the hair base labeled with $\alpha-E$ (1:200). Most gold grains are found over the extracellular sensillum lymph $(S L)$ between the microvilli and microlamellae of the auxiliary cells, but there is also some intracellular labeling in the tormogen cell (To). Part of another labeled sensillum is seen in the bottom right corner. Dendrites $(D)$ and epidermal cells $(E)$ are not labeled. The few grains found over the cuticle $(C)$ represent nonspecific background. $C$, Representative cross section through the distal portion of the third antennal segment after immunolabeling with $\alpha$-E (1:200). A sensillum trichodeum $(T)$ and three unspecified sensilla $(\bullet)$ are labeled, showing a high density of gold grains. A sensillum basiconicum $(B)$, a sensillum coeloconicum $(C o)$, and two unspecified sensilla $(O)$ are not labeled. The few scattered gold grains observed in nonlabeled areas represent nonspecific background. 


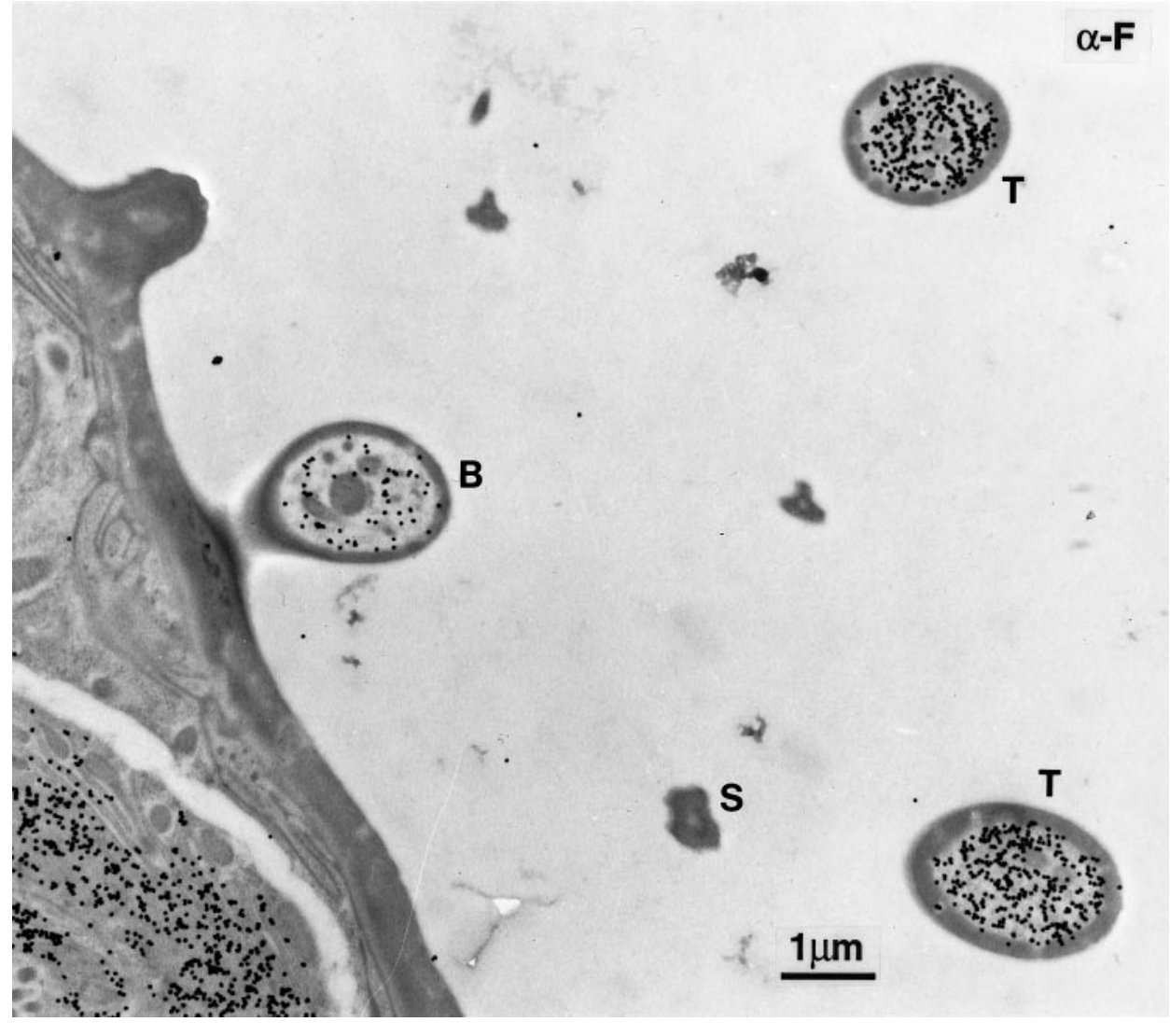

Figure 5. Representative immunocytochemical labeling of two classes of olfactory sensilla with subtracted antiserum against OS-E (1:60). Two sensilla trichodea $(T)$ are strongly labeled; a sensillum basiconicum $(B)$ shows weaker labeling (reduced density of gold grains). In the bottom left corner, part of another labeled sensillum is visible. $S$, Spinule (noninnervated microtrichium). compared with the sensilla basiconica, which display branched dendrites and a high pore density (Venkatesh and Singh, 1984; R. A. S., unpublished observations). However, on a single section this discrimination may not always be unequivocal, because the sensilla trichodea display progressively thinner walls and some dendritic branching toward the tip. Hence, accurate assessment of sensillar type requires a reconstruction of a series of sections through the same sensillum. Another potential complication is that intermediate sensillar types have been observed in other insect species and most likely occur in Drosophila as well (Steinbrecht, 1996a).

Sensilla that were labeled by anti-E or anti-F antibodies displayed the highest labeling density over the extracellular sensillum lymph in the lumen of the sensory hair as well as in the sensillum lymph cavity below the base of the hair (Fig. $4 A-C$ ). We did not observe labeling of either dendrites or cell bodies of olfactory receptor neurons. Some intracellular labeling was found in the sensillar auxiliary cells (Fig. 4B). Neither epidermal cells nor hemolymph was labeled. The cuticle often showed some nonspecific background, which also was observed when preimmune serum was substituted for affinity-purified antibody.

The immunolabeled sections revealed no differences between males and females, and the data from both sexes were, therefore, pooled. OS-E and OS-F were labeled in all typical sensilla trichodea $(n=102)$ and in 8 of 10 intermediate cases in which discrimination between sensilla trichodea and basiconica was uncertain. A fraction of the small sensilla basiconica, which are found intermingled between the sensilla trichodea on the ventrolateral portion of the antenna, also was labeled (15 of 34 sensilla examined). The labeling density on these sensilla was consistently lower than on the sensilla trichodea (Fig. 5). No labeling was observed in the large sensilla basiconica $(n=24)$, which are found primarily in the dorsomedial portion of the antenna, nor in hairs of another class, the sensilla coeloconica $(n=17)$.

\section{OS-E and OS-F colocalize in individual sensilla}

Antisera that are specific for OS-E or OS-F were produced by removing the fraction of one antiserum that bound to the second protein (cross-linked to beads), followed by affinity purification of the subtracted antiserum on beads containing the first protein (Fig. 6A). The antisera were specific for either OS-E or OS-F, respectively, on Western blots of extracts from bacteria expressing OS-E or OS-F (Fig. 6B). We noted the presence of faintly labeled dimer-sized bands of $\sim 30 \mathrm{kDa}$ in extracts prepared from bacteria expressing either OS-E or OS-F.

Using these subtracted affinity-purified antisera on alternating sections (Fig. 7A,B), we were able to check for the presence of both OS-E and OS-F in a series of individual sensilla. Whenever a sensillum was labeled by subtracted anti-OS-E, it also was labeled by anti-OS-F, and vice versa ( $n=113$ sensilla). There were also a number of sensilla that were not labeled by either antiserum, in particular those in the dorsomedial region of the antenna. The simplest interpretation of these results is that at least some individual sensilla contain more than one odorantbinding protein.

\section{DISCUSSION}

In this article we present evidence that $O S-E$ and $O S-F$, two members of a Drosophila family of genes that encode proteins structurally related to moth OBPs, are located $<1 \mathrm{~kb}$ apart and are coordinately expressed. We find that both OS-E and OS-F proteins are expressed in the same region of the Drosophila antenna and that both are present within two distinct classes of olfactory sensilla. Moreover, we consistently observe coexpression 
A

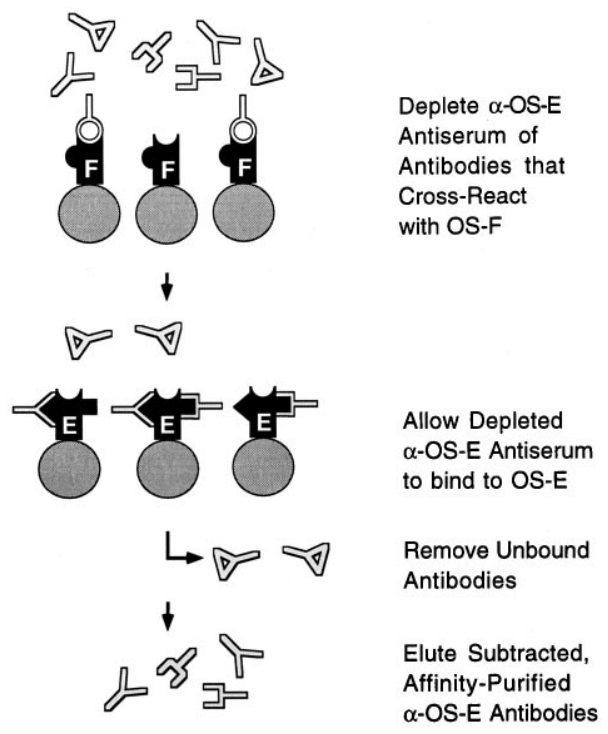

B

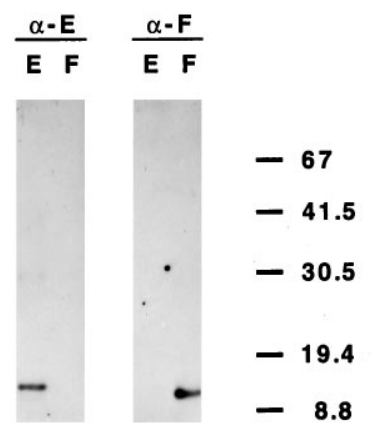

Figure 6. Subtracted anti-OS-E and anti-OS-F antisera are specific. $A$, Preparation of the subtracted anti-OS-E antiserum $(\alpha-E)$. (The reciprocal procedure was used to prepare subtracted anti-OS-F antiserum.) Briefly, the primary anti-OS-E antiserum was incubated with purified OS-F (conjugated to beads) to deplete it of antibodies that also recognize OS-F. Then the depleted anti-OS-E antiserum was affinity-purified by binding to purified OS-E (conjugated to beads) and then by subsequent elution. $B$, Western analysis showing that the subtracted anti-OS-E and anti-OS-F antisera are each specific for the corresponding purified protein. $0.1 \mu \mathrm{g}$ of OS-E $(E)$ and OS-F $(F)$ protein, purified from $E$. coli, were subjected to $15 \%$ SDS-PAGE and transferred to nitrocellulose. The subtracted affinity-purified $\alpha$-E (left) or $\alpha$-F (right) antiserum served as the primary antibody. Positions of molecular weight markers are indicated on the right. Note the presence of a faint dimer-sized band of $\sim 30 \mathrm{kDa}$ in the extreme left lane.

of OS-F in the same sensilla that express OS-E. This is the first demonstration of colocalization of two different OBPs within the same olfactory sensilla.

The localization of OS-E and OS-F proteins is consistent with the notion that they represent Drosophila homologs of moth OBPs. Both OS-E and OS-F are present in the sensillum lymph of sensory hairs housing olfactory receptor neurons as well as in the underlying auxiliary cells. Most likely, OS-E and OS-F are soluble proteins synthesized by the sensillar auxiliary cells, which then secrete them into the sensillar lymph surrounding the olfactory receptor neurons, as also is believed to be the case for the moth OBPs (Steinbrecht et al., 1992, 1995; Laue et al., 1994).

Expression of OS-E and OS-F is restricted to sensilla located in the ventrolateral region of the antenna. Within this region two sensillar types contain OS-E and OS-F proteins: most, if not all, sensilla trichodea and $\sim 40 \%$ of the interspersed small sensilla basiconica. We do not know whether the proteins have an identical function in the two sensillar types. However, expression of OS-E and OS-F may reflect a similar functional specialization of the sensilla that express them. In moths, different functional classes of sensilla express different classes of OBPs. The pheromone-binding proteins are associated with pheromonesensitive sensilla-primarily the specialized sensilla trichodea of the males (Steinbrecht et al., 1992)—whereas another group of OBPs, termed general odorant-binding proteins, is associated with sensilla that respond to general odorants-primarily the sensilla basiconica present in males and females (Laue et al., 1994; Steinbrecht et al., 1995). This distinction between PBP or GOBP expression seems to reflect a difference in sensillar function rather than simply a property of the morphological type. In Bombyx mori, PBPs are expressed in the sensilla trichodea of males, which respond to female pheromones, whereas GOBPs are expressed in the sensilla trichodea of females, which in this species respond to general plant odors (Steinbrecht et al., 1995).

The coexpression of different Drosophila OBPs (such as OS-E and OS-F) in subsets of sensory hairs could be important for the coding of olfactory information. Different moth OBPs can bind preferentially to distinct odorants (De Kramer and Hemberger, 1987; Du and Prestwich, 1995). Also, comparative immunocytochemical studies suggest a role for OBPs in olfactory coding (Steinbrecht, 1996b). Unlike the olfactory receptor cells of vertebrates, which project into a common OBP-filled mucus layer (Pevsner et al., 1988; Pevsner and Snyder, 1990), those of insects are compartmentalized into separate sensilla with distinct sensillum-lymph cavities. Expression of different combinations of OBPs within these various sensilla thus could influence which odorants have access to the enclosed olfactory receptor neurons. The coexpression of OS-E and OS-F within the same sensillum potentially could broaden the range of odorants to which the olfactory receptor neurons can respond. Different Drosophila sensilla may contain different combinations of the various OBP family members. In this regard, we note that the OS-E and OS-F proteins may not be the only OBPs expressed in the sensilla we examined. For example, there is at least one other OBP gene, PBPRP-1, the transcripts of which also are expressed in the ventrolateral region of the antenna (Pikielny et al., 1994) and which well may be expressed in a subset of the sensilla that express OS-E and OS-F. We note the formal possibility that the subtracted anti-E and anti-F antisera, which appear specific on Western blots (Fig. 6B) do cross-react to some degree in immuno-EM experiments. However, this is unlikely, because then we should have observed reciprocal differences in the intensity of the labeling of different hairs with the two antisera.

Another means of combinatorial coding suggested by our results is the possibility that OBPs coexpressed within a single sensillum could multimerize. We note that faint dimer-sized OS-E and OS-F bands are observed consistently in extracts prepared from bacteria that overexpress one or the other protein (Fig. $6 B$ ). If OS-E and OS-F are capable of dimerization, three distinct dimers (E-E, F-F, and E-F) and two monomers potentially could be present, each of which might have a different odorant speci- 


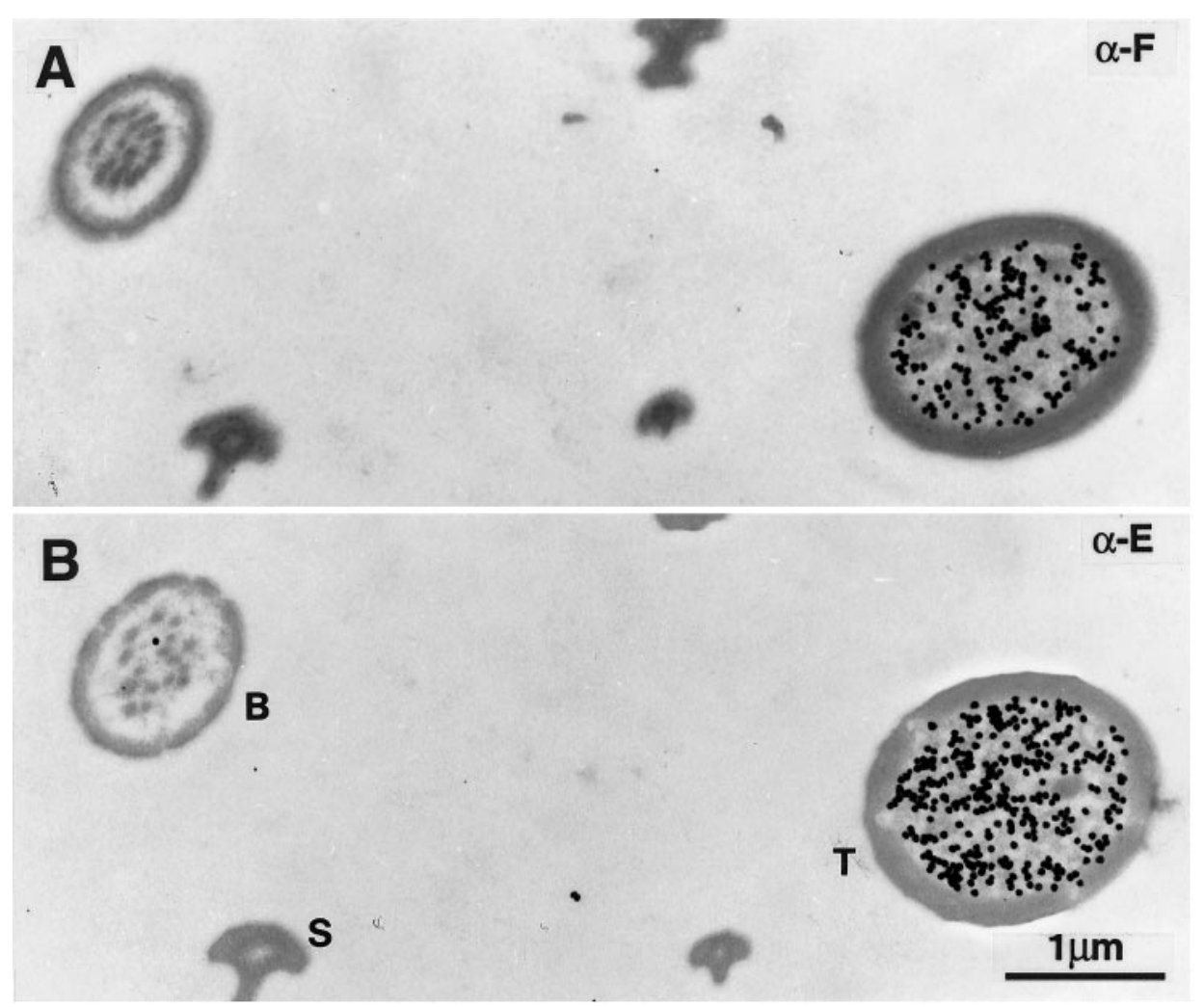

Figure 7. Immunocytochemical demonstration of OS-E and OS-F colocalization in olfactory hairs. $A$ and $B$ represent sections ( $\sim 0.4 \mu \mathrm{M}$ apart) of a section series through the same two sensilla. $A$ is labeled by subtracted $\alpha-F(1: 60)$, whereas $B$ is labeled by subtracted $\alpha-E(1: 60)$. In both sections, the sensillum trichodeum $(T)$ is strongly labeled, whereas the sensillum basiconicum $(B)$ remains unlabeled. $S$, Spinule. ficity. If different heterodimers transport different odorants to receptors, the possibility for heterodimer formation among a variety of OBPs that are expressed in overlapping sets of sensory hairs might provide a means of combinatorial coding of olfactory information.

The close proximity, similar sequences, common orientation, and shared intron positions of the $O S-E$ and $O S-F$ genes suggest that the two genes arose by a gene duplication event. The two additional introns in $O S-F$ might have arisen after such a duplication event. The coordinated expression of OS-E and OS-F proteins may result from the close proximity of the $O S-E$ and $O S-F$ genes. $O S-E$ and $O S-F$ likely share enhancer elements, some of which may have been duplicated during the initial expansion. The other presumptive Drosophila OBP genes are located at different chromosomal locations (Pikielny et al., 1994), and it is unknown whether their intron-exon organization resembles that of $O S-E$ and $O S-F$. In Caenorhabditis elegans (Troemel et al., 1995) and mouse (Sullivan et al., 1996), families of genes that encode chemosensory G-protein-coupled receptors are also present in clusters. However, genes within a single cluster are not regulated coordinately, because they are expressed in different chemosensory neurons (Troemel et al., 1995; Sullivan et al., 1996).

The close proximity of the $O S-E$ and $O S-F$ genes may present the opportunity to manipulate both genes simultaneously via a variety of genetic and molecular means (Ashburner, 1989). Electrophysiological or behavioral analyses of the odorant responses of the resulting Drosophila strains may be useful in addressing the function(s) of OS-E and OS-F proteins. It also may be possible to compare electrophysiologically the odorant responses of individual trichoid and basiconic sensilla that express altered levels of OS-E and OS-F via single-unit recordings (Siddiqi, 1987; P. Clyne and J. Carlson, unpublished data). Such experiments may provide additional insight into the roles of OBPs in olfactory signal transduction and chemosensory coding.

\section{REFERENCES}

Ashburner M (1989) Drosophila: a laboratory handbook. Cold Spring Harbor, NY: Cold Spring Harbor.

Breer H, Krieger J, Raming K (1990) A novel class of binding proteins in the antennae of the silk moth Antheraea pernyi. Insect Biochem 20:735-740.

Danscher G (1981) Localization of gold in biological tissue: a photochemical method for light and electron microscopy. Histochemistry 71:81-88.

De Kramer JJ, Hemberger J (1987) The neurobiology of pheromone reception. New York: Academic.

Du G, Prestwich GD (1995) Protein structure encodes the ligand-binding specificity in pheromone-binding proteins. Biochemistry 34:8726-8732.

Farbman A (1992) Cell biology of olfaction. Cambridge, UK: Cambridge UP.

Feinberg A, Vogelstein B (1983) A technique for radiolabelling DNA restriction endonuclease fragments to high specific activity. Anal Biochem 132:6-13.

Harlow E, Lane D (1988) Antibodies: a laboratory manual. Cold Spring Harbor, NY: Cold Spring Harbor.

Helfand SH, Carlson JR (1989) Isolation and characterization of an olfactory mutant in Drosophila with a chemically specific defect. Proc Natl Acad Sci USA 86:2908-2912.

Hildebrand JG (1995) Analysis of chemical signals by nervous systems. Proc Natl Acad Sci USA 92:67-74.

Hung M-C, Wensink PC (1984) Different restriction enzyme-generated sticky DNA ends joined in vitro. Nucleic Acids Res 12:1863-1874.

Laue M, Steinbrecht RA, Ziegelberger G (1994) Immunocytochemical localization of general odorant-binding protein in olfactory sensilla of the silkmoth Antheraea polyphemus. Naturwissenschaften 81:178-180.

McKenna MP, Hekmat-Scafe DS, Gaines P, Carlson JR (1994) Putative Drosophila pheromone-binding proteins expressed in a subregion of the olfactory system. J Biol Chem 269:16340-16347.

Nonet ML, Grundahl K, Meyer BJ, Rand JB (1993) Synaptic function is impaired but not eliminated in C. elegans mutants lacking synaptotagmin. Cell 73:1291-1305. 
Pelosi P (1994) Odorant-binding proteins. Crit Rev Biochem Mol Biol 29:199-228.

Pelosi P, Maida R (1995) Odorant-binding proteins in insects. Comp Biochem Physiol [B] 111:503-514.

Pevsner J, Snyder SH (1990) Odorant-binding protein: odorant transport function in the vertebrate nasal epithelium. Chem Senses 15:217-222.

Pevsner J, Hwang PM, Sklar PB, Venable JC, Snyder SH (1988) Odorantbinding protein and its mRNA are localized to lateral nasal gland implying a carrier function. Proc Natl Acad Sci USA 85:2383-2387.

Pierce JC, Sternberg NL (1992) Using bacteriophage P1 system to clone high molecular weight genomic DNA. Methods Enzymol 216:549-574.

Pikielny CW, Hasan G, Rouyer F, Rosbash M (1994) Members of a family of Drosophila putative odorant-binding proteins are expressed in different subsets of olfactory hairs. Neuron 12:35-49.

Prestwich GD (1993) Bacterial expression and photoaffinity labelling of a pheromone binding protein. Protein Sci 2:420-428.

Prestwich GD, Du G, LaForest S (1995) How is pheromone specificity encoded in proteins? Chem Senses 20:461-469.

Raha D, Carlson J (1994) OS9: a novel olfactory gene of Drosophila expressed in two olfactory organs. J Neurobiol 25:169-184.

Sambrook J, Fritsch EF, Maniatis T (1989) Molecular cloning: a laboratory manual. Cold Spring Harbor, NY: Cold Spring Harbor.

Siddiqi O (1987) Neurogenetics of olfaction in Drosophila melanogaster. Trends Genet 3:137-142.

Smoller DA, Petrov D, Hartl DL (1991) Characterization of bacteriophage P1 library containing inserts of Drosophila DNA of 75-100 kilobase pairs. Chromosoma 100:487-494.

Steinbrecht RA (1993) Freeze-substitution for morphological and immunocytochemical studies in insects. Microsc Res Tech 24:488-504.

Steinbrecht RA (1996a) Structure and function of insect olfactory sen- silla. In: Olfaction in mosquito-host interactions (Bock GR, Cardew G, eds), pp 158-177. Chichester, UK: Wiley.

Steinbrecht RA (1996) Are odorant-binding proteins involved in odorant discrimination? Chem Senses 21:719-727.

Steinbrecht RA, Ozaki M, Ziegelberger G (1992) Immunocytochemical localization of pheromone-binding protein in moth antennae. Cell Tissue Res 270:287-302.

Steinbrecht RA, Laue M, Ziegelberger G (1995) Immunolocalization of pheromone-binding and general odorant-binding protein in olfactory sensilla of the silkmoths Antheraea and Bombyx. Cell Tissue Res 282:203-217.

Stocker RF (1994) The organization of the chemosensory system in Drosophila melanogaster: a review. Cell Tissue Res 275:3-26.

Störtkuhl KF, Hofbauer A, Keller V, Gendre N, Stocker RF (1994) Analysis of immunocytochemical staining patterns in the antennal system of Drosophila melanogaster. Cell Tissue Res 275:27-38.

Sullivan SL, Adamson MC, Ressler KJ, Kozak CA, Buck LB (1996) The chromosomal distribution of mouse odorant receptor genes. Proc Natl Acad Sci USA 93:884-888.

Troemel ER, Chou JH, Dwyer ND, Colbert HA, Bargmann CI (1995) Divergent seven transmembrane receptors are candidate chemosensory receptors in C. elegans. Cell 83:207-218.

Venkatesh S, Singh R (1984) Sensilla on the third antennal segment of Drosophila melanogaster meigen. Int $\mathrm{J}$ Insect Morphol Embryol 13:51-63.

Vogt RG, Riddiford LM (1981) Pheromone binding and inactivation by moth antennae. Nature 293:161-163.

Vogt RG, Prestwich GD, Lerner MR (1991) Odorant-binding-protein subfamilies associate with distinct classes of olfactory receptor neurons in insects. J Neurobiol 22:74-84. 